Diagnóstico de estrés por sobrecarga basado en el modelo conceptual de Imogene King

\title{
Autoras:
}

Natalia Pérez Ortiz. Estudiante de la Licenciatura en Enfermería. Universidad de Costa Rica.

Vivian Vílchez-Barboza. Enfermera, Doctora en Enfermería. Docente Escuela de Enfermería. Universidad de Costa Rica. E-mail: vivian.vilchez@ucr.ac.cr

* Proceso de enfermería realizado durante el desarrollo del Trabajo Final de Graduación titulado: "Efecto de Intervención de Enfermería en Riesgos Psicosociales del Trabajo para la Calidad de Vida Relacionada con Salud en Funcionarios(as) de una Institución Pública Costarricense, durante el año 2016", como requisito para optar por el grado de Licenciatura en Enfermería de la Universidad de Costa Rica.

\section{> Resumen}

El presente estudio de caso se realizó en un adulto trabajador de una institución pública costarricense. Se utilizó el Modelo Conceptual de Interacción de Sistemas de Imogene King y su teoría de Consecución de Objetivos. La metodología establecida para el estudio de caso fue a través de la aplicación del Proceso de Enfermería y la utilización del lenguaje taxonómico como indicador empírico para la planificación del cuidado; NANDA en la expresión de diagnósticos, NOC para la Clasificación de los Resultados de Enfermería (NOC) y NIC para la Clasificación de Intervenciones de Enfermería (NIC). Se desarrolló un plan de cuidados priorizando las necesidades, con intervenciones que dieron respuesta a dichas necesidades. Como resultado se obtuvo la evidencia de la gestión del cuidado de enfermería en un ambiente laboral, a una persona con diagnóstico de estrés por sobrecarga.

> Palabras clave: Enfermería, Atención de Enfermería, Calidad de Vida, Salud Laboral, Riesgos Laborales (DeCS). 


\section{Diagnosis of stress overload} based on Imogene King's conceptual model

\section{> Abstract}

This case study was conducted with an adult worker in a public institution of Costarrica. We used the Imogene King's Conceptual Model of Systems Interaction and her Achievement Goal Theory. The established methodology is a case study that developed the Nursing Process and the use of taxonomic language as an empirical indicator for care planning; we applied NANDA for the diagnosis expressions, we also used NOC for the Nursing Outcomes Classification (NOC) and NIC for the Nursing Interventions Classification (NIC). A care plan was developed prioritizing the needs, it includes interventions that answered those needs. As a result, we got the evidence of Nursing care management in a work environment of a person with stress overload diagnosis.

> Keywords: Nursing, Nursing Care, Occupational Health, Life quality, Occupational (DeCS). 


\section{, Introducción}

En el transcurso del siglo $X X$ se ha producido un cambio en el concepto de salud como un constructo social. Definido como un fenómeno con implicaciones sociales, políticas, económicas, y culturales, pero sobre todo un derecho fundamental (1). King agrega que, salud es un proceso dinámico del ciclo vital, el cual contempla los ajustes a los agentes estresantes del entorno interno y externo, ajustes que se realizan a través de la optimización del uso de los recursos para lograr el máximo potencial para la vida diaria y que influyen en la capacidad para funcionar en los roles sociales (2). Aunado a esto, es evidente que trabajar va siendo cada vez menos una actividad fisica y pasa a ser sobre todo una actividad mental, de procesamiento de información, de solución de problemas y de gestión de incertidumbre (3).

Por tanto, se da relevancia a aspectos como la iniciativa, la toma de decisiones, la asunción de riesgos para resolver problemas, la innovación en el puesto de trabajo, los mecanismos de autocontrol y autorregulación y el desarrollo de estrategias de automotivación (3); dando respuesta a este contexto, es que se establecen políticas dirigidas a la búsqueda de entornos laborales saludables, donde se incluye la prevención de riesgos ergonómicos y psicosociales derivado de las tareas, la tecnología y las personas.

En concordancia con lo mencionado, la Calidad de Vida Relacionada con Salud (CVRS) se convierte en un constructo relevante a trabajar por la enfermería, la cual es comprendida, como la percepción que tiene el individuo de su estado de salud (4), en la cual el ambiente laboral es un factor altamente influyente, por el tiempo y esfuerzo que cada persona le invierte.

De acuerdo con esto, el plan de estudios de Licen- ciatura en Enfermería de la Universidad de Costa Rica, insta a trabajar la salud no solo en ámbitos hospitalarios, sino también extrahospitalarios, como lo son los ambientes laborales, considerando la importancia de promocionar la salud y dar respuesta desde la disciplina al contexto epidemiológico del país. Es por esta razón, que se desarrolla este Trabajo Final de Graduación, titulado: "Efecto de Intervención de Enfermería en Riesgos Psicosociales del Trabajo para la Calidad de Vida Relacionada con Salud en Funcionarios(as) de una Institución Pública Costarricense, durante el año 2016", que consiste en sistematizar e integrar conocimientos alrededor de un tema académico, incorporando la teoría propia de la disciplina y un método de investigación científico validado. Para lograr un análisis sobre el tema, este trabajo posee dos componentes: uno investigativo y uno reflexivo, el presente manuscrito corresponde a la fase reflexiva.

El abordaje consistió en la aplicación del Proceso de Enfermería (PE), entendido como la aplicación del método científico en la práctica asistencial que nos permite a los/as profesionales de Enfermería prestar cuidados que se nos demandan, de una forma estructurada, homogénea, lógica y sistemática (5).

Así, el objetivo de este estudio de caso es describir el proceso de enfermería aplicado a un adulto trabajador con necesidad de disminuir el estrés por sobrecarga laboral, por medio una intervención de enfermería.

\section{> Metodología}

Un estudio de caso, es una herramienta valiosa de investigación que permite medir y registrar la conducta de la persona involucrada en el fenómeno estudiado (6). Para la etapa de valoración, se realizó un análisis desde la teoría de Imogene King, 
bajo el marco conceptual del Sistema de Interacciones (7).

La teoría sustenta que el Proceso de cuidado de Enfermería identifica y explica algunas de las funciones esenciales de los/as enfermeros/as, que son la evaluación de salud de las personas, la formulación de un plan de acción y la evaluación de su efectividad (8). Para el diagnóstico del caso, se establece el uso de la Taxonomía NANDA I 20152017 conformada por 13 dominios, 47 clases y 235 etiquetas diagnósticas (9), y sus respectivos vínculos con NOC (Nursing Results) y NIC (Nursing Interventions).

El Proceso de Enfermería se realizó en un período de cuatro meses distribuidos de la siguiente manera:

- Mes 1: Un primer momento donde se ejecuta la valoración, seguido de un análisis crítico y reflexivo de la información obtenida para la identificación de la necesidad prioritaria, diagnóstico con taxonomía NANDA, NIC y NOC para el desarrollo del plan de cuidados.

- Mes 2: En un segundo momento, se comenta el diagnóstico con el usuario y se establece un acuerdo para realizar las actividades propuestas considerando la disponibilidad de tiempo.

- Mes 3: La tercera sesión consistió en el seguimiento al usuario, donde se ve la evolución en la ejecución de las actividades y se identifican los posibles obstáculos percibidos y sentimientos experimentados.

- Mes 4: En la cuarta sesión, se evaluó a la persona y se aplicaron los instrumentos iniciales en una segunda medición.

La valoración incluyó la entrevista del Historial de Enfermería (10), el cual se compone de los instrumentos: a) Diabetes, Hipertensión y Factores de Riesgo de Enfermedades Crónicas, este cuestionario fue validado en la encuesta basal del programa Conjunto de Acciones para la Reducción Multifactorial de Enfermedades No transmisibles (CARMEN) que se realizó en el año 2000, en el Cantón Central de Cartago (11).

b) Cuestionario SF-36 v.2, que mide Calidad de Vida relacionada con Salud (CVRS), este instrumento es considerado el mejor para medir el estado de salud de una población desde un punto de vista global; contiene 36 ítems e incluye 8 dimensiones: función física, rol físico, dolor corporal, salud general, vitalidad, función social, rol emocional y salud mental. Las cuales se resumen en dos componentes: Salud Física y Salud Mental (12).

c) Además, se realizó una valoración física que incluyó la toma de presión arterial, frecuencia cardíaca, respiraciones por minuto, temperatura corporal, y medidas antropométricas como el peso, talla e índice de masa corporal (IMC).

\section{> Descripción del caso}

L.X.W (como se denominará en adelante al usuario), es un hombre costarricense, de 28 años de edad, casado sin hijos. Trabaja como abogado, brindando sus servicios a una institución pública, con una jornada laboral entre 10 a 12 horas por día, incluidas las que utiliza en su casa, además los fines de semana suele trabajar.

\section{VALORACIÓN GENERAL}

La primera etapa del Proceso de Enfermería es la valoración, donde es importante destacar que debe ser integral y eficaz (13).

Los datos en la valoración individual de L.X.W son 
los siguientes: como antecedentes tiene Hipertensión Arterial (HTA) desde hace un año (27 años), controla la patología con medicamentos como Enalapril (14) e Hidroclorotiazida (15), además de ir a control cada 6 meses.

En la valoración física, se obtuvieron los siguientes valores en los signos vitales: Presión arterial: 118/80 mmHg, frecuencia cardíaca 62 latidos/minuto, frecuencia respiratoria 16 respiraciones/minuto, temperatura $36,2^{\circ} \mathrm{C}$, talla 1,64 metros, peso $84 \mathrm{Kg}$ y un IMC de 31,23 , que se categoriza como obesidad moderada (16).

Para la descripción del Historial de Enfermería, se desarrollan cada uno de los sistemas del usuario:

- Sistema personal: Dentro de la percepción y conocimiento del "yo", L.X.W señala que "se siente perdido, que ha perdido su forma de ser por el estrés del trabajo". Este estrés, manifiesta, le ha generado muchos problemas físicos, ha notado que en épocas de alta carga laboral, la presión arterial se ve afectada, le genera dolores corporales característicos del estrés, como la tensión muscular en hombros, espalda, cefaleas, colitis, entre otros (17). Señala que padecer a tan temprana edad HTA, le genera preocupación y temor porque cree que su salud va a empeorar. Además, es su trabajo siente frustración, según sus propias palabras "quisiera salir corriendo".

- Sistema interpersonal: El subsistema conyugal, se ha visto afectado por la demanda laboral y el tiempo que exige, L.X.W expresó que su esposa se ha sentido "sola", creé que no le dedica tiempo suficiente a la pareja y esto le ha traído problemas que generan más ansiedad en el usuario.

- Sistema social: En relación al sistema social, L.X.W señala que siente una carga de trabajo importante, su principal molestia se engloba en la cantidad de trabajo y tiempo para presentarlo, esto le ha generado una insatisfacción general en cada aspecto del trabajo: supervisión, ambiente, prestaciones recibidas y en la parte intrínseca.

\section{ETAPA DIAGNÓSTICA}

Corresponde a la segunda fase del Proceso de Enfermería, y se refiere al juicio clínico sobre las respuestas individuales, familiares o de la comunidad a problemas de salud vitales reales o potenciales, que requiera de la intervención de enfermería con el objeto de resolverlo o disminuirlo (18).

Para la aplicación de la etapa diagnóstica, se utilizó la taxonomía propuesta por NANDA-I (North American Nursing Diagnostics Association) (19). El diagnóstico prioritario pertenece al dominio de Afrontamiento/Tolerancia del estrés (20), enfocado en promover la salud laboral y reducir riesgos psicosociales, generando un impacto positivo para el sistema personal, interpersonal y social del usuario. El diagnóstico seleccionado fue:

\section{Estrés por sobrecarga}

Dentro de las características definitorias que se encontraron en la valoración, L.X.W presenta la expresión del exceso de estrés situacional y la sensación de tensión (21).

\section{ETAPA DE PLANIFICACIÓN}

La etapa de planificación se sustentó en las acciones de enfermería basada en las respuestas humanas (22), abordadas desde la teoría de Consecución de Objetivos, propuesta por Imogene King (23). En esta etapa se busca la formulación de objetivos de independencia en base a las causas de dificultad detectadas, además se procede a la elección del modo de intervención y la determinación de actividades que impliquen a la persona en 
su propio cuidado (24).

Se identifican como NOC (25) los siguientes resultados para medir la resolución del diagnóstico: (1212) Niveles de estrés y (2102) Nivel de dolor, donde se establece el siguiente objetivo para alcanzar en conjunto con el usuario: El usuario será capaz de reducir el estrés por sobrecarga en un período de 4 meses. Para dichos NOC, se establecen los siguientes indicadores:

(1212) Nivel de estrés:

(121201) Aumento de la presión arterial

(121205) Aumento de la tensión muscular en hombros

(121206) Cefalea tensional

(121212) Malestar estomacal

(121222) Ansiedad

(2102) Nivel de dolor:

(210201) Dolor referido

(210209) Tensión muscular

(210212) Presión arterial

La intervención (NIC) (26) que se planificó valorando la relevancia de: (0180) Manejo de la energía y (1400) Manejo del dolor, donde se plantean las siguientes actividades:

\section{EJECUCIÓN DE ACTIVIDADES}

La penúltima etapa consta de la aplicación del plan de cuidado, que desarrolla tres criterios: preparación, la ejecución propiamente dicha y la documentación o registro, donde interviene según la planificación, la persona, el/la enfermero/a, el equipo de salud, los familiares y las redes de apoyo, con la dirección del profesional de Enfermería (27).

Para el desarrollo de esta etapa, se llevó a cabo una actividad que incluyó la organización de prioridades en la vida, tomando en cuenta que las per- sonas son seres integrales (28). Se diseñaron dos tablas iguales donde se colocaron tres cuadros en cada una, acomodados en orden jerárquico como primer, segundo y tercer nivel; en la primera tabla se le pidió al usuario que escribiera en orden las tres prioridades actuales en su vida, el resultado fue el siguiente: $1^{\circ}$ Trabajo, $2^{\circ}$ Familia y $3^{\circ}$ Él mismo. Seguidamente, se le solicitó que hiciera un breve análisis de la tabla y reorganizara las prioridades según lo que consideraba que era realmente importante, en la segunda tabla se obtuvo lo siguiente: $1^{\circ}$ Él mismo, $2^{\circ}$ Familia y $3^{\circ}$ Trabajo.

Además, se incluyó una hoja donde se encontraba la figura de una persona, el usuario marcó las zonas de dolor y se realizó la respectiva explicación sobre la relación entre el estrés manifestado y las zonas afectadas (29). Finalmente, después de la reorganización y la elección de las zonas de dolor, se llegó a un objetivo común que recibió seguimiento mensual durante cuatro meses.

\section{EVALUACIÓN DE RESULTADOS}

La última etapa del proceso es la evaluación, definida como "una actividad planificada, en curso, con propósito, en el que los/as usuarios/as y los/ as profesionales de la salud determinan el progreso del/la usuario/a hacia el logro de metas / resultados y la eficacia del plan de cuidados de enfermería" (30).

Para la evaluación y el alcance de los objetivos planteados, se desarrolló un proceso de cuatro meses con citas periódicas cada mes. En la primera cita se realizó la entrega del diagnóstico de Enfermería, posterior al correspondiente análisis de los resultados obtenidos en el instrumento SF-36, y la organización de las actividades antes descritas; en la segunda cita se llevó a cabo un seguimiento, donde se abarcaron las siguientes preguntas: ¿Cómo ha sido llevar el proceso y cumplir 
con las actividades?, ¿Cuáles han sido los principales obstáculos para alcanzar la meta?, ¿Existe un punto que deseé agregar al proceso para establecer como objetivo?; para cada pregunta el usuario brindaba su respuesta y se llegó al acuerdo de incluir en la meta la mejora en la condición física a través del ejercicio y la asesoría nutricional. La cita final fue donde se realizó la evaluación y se volvió a aplicar el instrumento SF-36 para evaluar los logros alcanzados durante el proceso.

Como resultado de esta evaluación, se obtuvieron los siguientes resultados:

- Componente de Salud Física: En la primera medición (Mes 1) el puntaje obtenido en este componente fue 61 y en la segunda medición (Mes 2) el puntaje fue 82.

A continuación, en el Gráfico 1 se muestran los puntajes obtenidos en cada una de las dimensiones que integran el Componente de Salud Física:

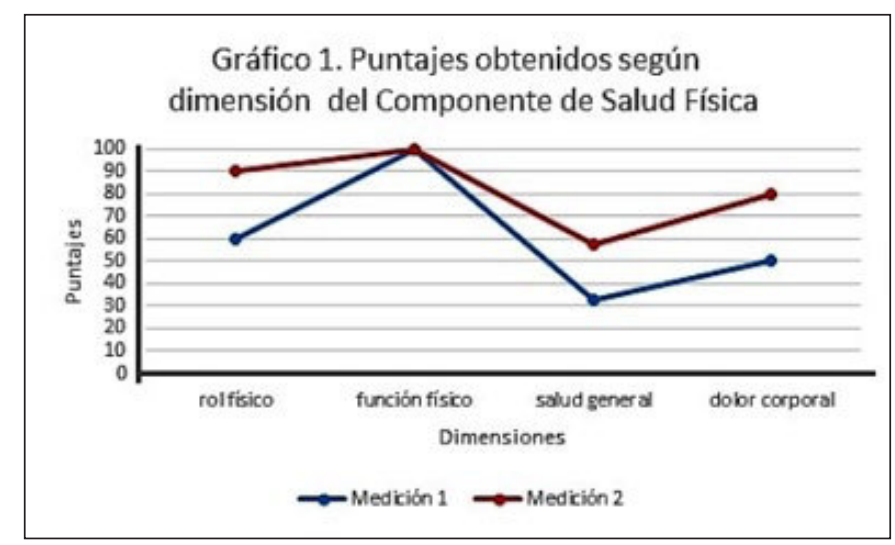

Fuente: Elaboración propia.

- Componente de Salud Mental: En la primera medición (Mes 1) el puntaje obtenido en este componente fue 67 y en la segunda medición (Mes 2) el puntaje fue 76 .

A continuación, en el Gráfico 2, se muestran los puntajes obtenidos en cada una de las dimensiones que integran el Componente de Salud Mental:

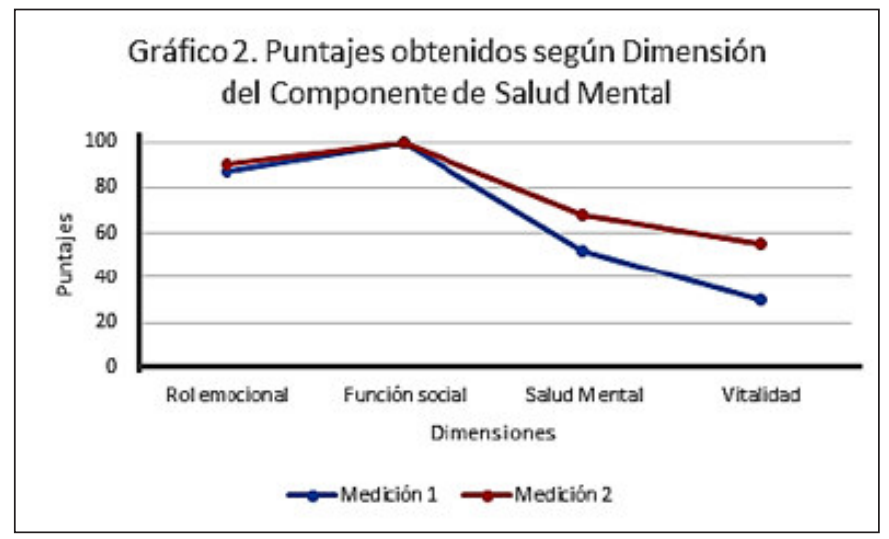

Fuente: Elaboración propia.

Como se observa, cada uno de los componentes con sus dimensiones aumentaron el puntaje en cuanto a la CVRS, lo cual fue congruente con lo expresado por el trabajador. Los indicadores planteados para la evaluación alcanzaron puntajes que evidenciaron el alcance de los objetivos establecidos en el plan de cuidados. El trabajador manifestó sentirse mucho mejor, haber recuperado su estabilidad de pareja y parte de su esencia como persona.

\section{) Conclusiones}

El desarrollo del presente estudio de caso concluye que la presencia de Enfermería en un espacio laboral desarrolla una mejoría en la salud de las personas trabajadoras. Se destaca, según lo visto en la etapa de evaluación, que un proceso de cuatro meses conlleva una mejoría importante, sin embargo, aún existen dimensiones como "Salud general" y "Vitalidad" que continúan con una nota por debajo de los rangos adecuados; esto refleja la necesidad de brindar continuidad al proceso dentro del área de trabajo, para promover el aumento de cada una de las dimensiones, siempre enfocado en las respuestas humanas individuales que generan necesidades de abordaje personalizadas. El o la enfermero/a es capaz de llevar a cabo dicho seguimiento, promoviendo el alcance 
de metas, no sólo a nivel individual, sino grupal, por el impacto que tiene un trabajador con un estado adecuado de salud y motivado, en la productividad de la institución.

\section{Referencias bibliográficas}

1. Organización Panamericana de la Salud, Organización Mundial de la Salud. Renovación de la atención primaria de salud en las américas. Documento de posición de la Organización Panamericana de la Salud/Organización Mundial de la Salud (OPS/OMS). Washington (EEUU): OPS; 2005. 33p.

2. King IM. A theory for nursing: System, concepts, process. New York (EEUU): John Wiley \& Sons. 1981. 181p.

3. Peiró JM. El Sistema de Trabajo y sus implicaciones para la prevención de los riesgos psicosociales en el trabajo. Univ Psychol. 2004; 3(2): $179-86$

4. García GH, Vera G, Lugo L. Calidad de vida relacionada con la salud en Medellín y su área metropolitana, con aplicación del SF-36. Revista Facultad Nacional de Salud Pública. 2013; 3(21): 2639.

5. Burgos M, Paravic T. Enfermería como profesión. Rev Cubana Enfermer. [en línea] 2009. [acceso jun 2016] 25(1-2). Disponible en: http://scielo. sld.cu/pdf/enf/v25n1-2/enf101_209.pdf.

6. Martínez PC. El método de estudio de caso: estrategia metodológica de la investigación científica. Pensamiento y Gestión. 2006; 20:165-93.

7. Rachal B. Imogene King: King's conceptual systems and theory of goal attainment. 2013. Disponible en https:/ / brandonrachaldotme.files.wordpress.com/2013/01/ imogene-king-handout.pdf
8. Raile M, Marriner A. Modelos y teorías en enfermería. 7ma ed. Barcelona (España): Elsevier / Mosby. 2011. 797p.

9. Caballero E, Becerra R, Hullin C. Proceso de Enfermería e informática para la gestión del cuidado. Santiago de Chile: Editorial Mediterráneo; 2010. 414p.

10. Figueroa GJ, Rodríguez V. Proceso de Enfermería. Universidad del Sagrado Corazón. [en línea] 2010. [acceso jun 2016] Disponible en: http:// handbook.usfx. bo/nueva/vicerrectorado/citas/ SALUD_10/Enfermeria_Obtetriz/19.pdf

11. Organización Panamericana de la Salud (OPS). Encuesta de Diabetes, Hipertensión y Factores de Riesgo de Enfermedades Crónicas. Washington (EEUU): OPS; 2010. 176p.

12. Tuesca R. La Calidad de vida, su importancia y cómo medirla. Salud Uninorte. 2005; 21: 76-86.

13. Duque P. Factores relacionados con la aplicabilidad del proceso atención de enfermería en una institución de salud de alta complejidad en la ciudad de Medellín, Colombia. Rev Univ Salud. [en línea]. 2014 91-102; 2014. Disponible en: http:// www.scielo.org.co/ pdf/reus/v16n1/v16n1a09.pdf

14. Fernández P, Lizasoain I, Leza JC, Moreno A. Manual de farmacología básica y clínica. 18va ed. Editorial Médica Panamericana. Madrid (España).2012. 1344p.

15. Katzung B, Masters S, Trevor A. Farmacología básica y clínica. 12ma ed. McGraw-Hill. México DF (México). 2013. 1236p.

16. Lopategui E. Determinación del Índice de Masa Corporal. Experimento de laboratorio I-23 [en línea]. 2008. [acceso jun 2016]; Disponible en: http://www.saludmed.com/labsfisiologiaejercicio/nutricionyantropometricas/LAB_I23-Indice_ Masa_ Corporal.pdf 
17. Daneri MF. Psicobiología del Estrés. Faculta de Psicología Universidad de Buenos Aires. [en línea]. 2012. [acceso jun 2016]; Disponible en: http://www.psi.uba.ar/academica/carrerasdegrado/psicologia/sitios_catedras/electivas/090_comportamiento/material/tp_estres. pdf

18. Ministerio de Salud Pública y Bienestar Social, Instituto Nacional de Educación Permanente en Enfermería y Obstetricia (INEPEO). Proceso de Atención de Enfermería (PAE). Rev Salud Pública Parag. 2013; 3(1): 41-8.

19. NANDA Internacional. Diagnósticos enfermeros. Definición y clasificación 2015-2017. Barcelona (España): Elsevier; 2014. 512p.

20. Barreto P, De la Torre O, Pérez-Marín M. Detección del duelo complicado. Psicooncología. 2012; 9(2-3); 355-68.

21. Mingote JC, Pérez S. Estrés en la Enfermería. El cuidado del cuidador. Madrid (España): Ediciones Díaz de Santos; 2003. 160p.

22. Gobernado MM. El proceso del duelo; prevención y tratamiento de su complicación. [Tesis de pregrado] Murcia (España): Universidad Católica de Murcia; 2014.

23. Salazar Y. Proceso de Atención de Enfermería P.A.E. Universidad Nacional Autónoma de México. [en línea]. 2013. [acceso jun 2016]; Disponible en: http://www.eneo.unam.mx/publicaciones/publicacionesoct2013/Proceso\%20atencion\%20en\%20Enfermeria.pdf

24. Moorhead S, Johnson ML, Maas M, Swanson E. Clasificación de resultados de enfermería (NOC): Medición de resultados en salud. 4ta ed. Madrid (España): Elsevier; 2009. 912p.
25. Bulechek G, Butcher H., McCloskey- Dochterm J. Clasificación de intervenciones de Enfermería (NIC). 5ta ed. Madrid (España): Elsevier; 2010. 976p.

26. Fernández C. M. La psicoterapia cognitivo conductual y algunas terapias alternativas complementarias (Propuesta de intervención). [Tesis de pregrado] Estado de México: Universidad autónoma del Estado de México; 2013.

27. Martínez M. Dimensiones básicas de un Desarrollo Humano Integral. Rev Univ Bolivariana. 2009; 8(23): 119-38.

28. Comín E. Albarrám I. Gracia A. El estrés y el riesgo para la salud. Zaragoza (España): Editorial MAZ; 2015. 46p.

29. Doenges E. Moorhouse MF. Proceso y diagnósticos de enfermería: aplicaciones: aplicaciones. México D.F: Editorial El Manual Moderno; 2014. $319 p$.

30. Alfaro P, Chaves N, Mata K, Pérez N, Vílchez V. Proceso de Enfermería en un adulto trabajador con diagnóstico de duelo complicado. Sanus. [en línea] 2016 [acceso oct 2016]; 1(2): 38-45. Disponible en: http://www.sanus.uson.mx/resumen. php?rev $=2 \& \operatorname{art}=14$ 